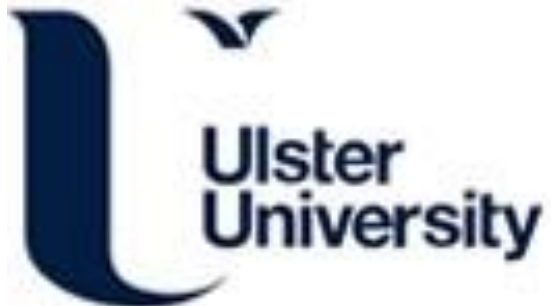

\section{Applications, composites, and devices: general discussion}

Bikkarolla, S., Baxendale, M., Ewels, C., Enoki, T., Kaneko, K., Martın, N., Santa-Cruz, P., Edwards, R. Khanam, Z., Zitoun, D., Ajayan, P., Khare, V., Zopfl, A., Gspann, T., Ogihara, H., Shaffer, M., Coleman, K., Chan-Park, M., Papakonstantinou, P., ... Kinloch, I. (2014). Applications, composites, and devices: general discussion. Faraday Discussions, 2014, 429-443. https://doi.org/10.1039/C4FD90046D

Link to publication record in Ulster University Research Portal

Published in:

Faraday Discussions

Publication Status:

Published (in print/issue): 18/11/2014

DOI:

10.1039/C4FD90046D

\section{Document Version}

Publisher's PDF, also known as Version of record

\section{General rights}

Copyright for the publications made accessible via Ulster University's Research Portal is retained by the author(s) and / or other copyright owners and it is a condition of accessing these publications that users recognise and abide by the legal requirements associated with these rights.

\section{Take down policy}

The Research Portal is Ulster University's institutional repository that provides access to Ulster's research outputs. Every effort has been made to ensure that content in the Research Portal does not infringe any person's rights, or applicable UK laws. If you discover content in the Research Portal that you believe breaches copyright or violates any law, please contact pure-support@ulster.ac.uk. 


\section{Applications, composites, and devices: general discussion}

Santosh Kumar Bikkarolla, Mark Baxendale, Chris Ewels, Toshiaki Enoki, Katsumi Kaneko, Nazario Martín, Petrus Santa-Cruz, Rebecca Edwards, Zeba Khanam, David Zitoun, Pulickel Ajayan, Varsha Khare, Alexander Zöpfl, Thurid Gspann, Hitoshi Ogihara, Milo Shaffer, Karl Coleman, Mary Chan-Park, Pagona Papakonstantinou, Sehmus Ozden, Oana Andreea Bârsan, Alan Windle and Ian Kinloch

DOI: 10.1039/C4FD90046D

Karl Coleman opened the discussion of the paper by Mary Chan-Park: Is the source of the SWCNTs important? I ask this in the context that the ratio of SC to metallic will be different from different suppliers and methods of production.

Mary Chan-Park responded: We have not tried other suppliers of nanotubes but in the literature, gel electrophoresis sorting has been applied to HiPco ${ }^{\circledR}$ and CoMoCAT ${ }^{\circledR}$ tubes. For the radical preferential attack, the diameter effect is important.

Karl Coleman commented: During the electrophoresis does the source of the NTs effect the movement/separation on the gel.

Mary Chan-Park answered: The different types can mostly work, and the small diameter tubes are easier to separate than the large diameter tubes in general.

Thurid Gspann said: There appears to be a length issue when dealing with carbon nanotubes. On the one hand for most applications, mechanical as well as electrical, CNT ends represent the ultimate defect. In other words CNTs should be as long as possible. On the other hand, purification, solubilisation and separation processes either shorten CNTs or require them to be of a length usually less than 5 micron. What is the estimated maximum length of CNTs to be separated by gel electrophoresis? And do you see any possibilities of increasing this length to say 100 micron range?

Mary Chan-Park responded: I think the length of tubes separated by gel electrophoresis is around 0.5 micron but the measured lengths vary from 300 to 800 $\mathrm{nm}$ (see Fig. 5 in my manuscript). 
Mark Baxendale remarked: Are the channels of your FETs made from individual semiconductor nanotubes, networks comprising only semiconductor nanotubes, or networks comprising both semiconductors and a small population of metallic nanotubes?

Mary Chan-Park answered: The channels are networks of mainly semiconducting tubes but with small amounts of metallic tubes.

Mark Baxendale asked: Is it possible to say whether the FET response is that due to the intrinsic properties of the constituent nanotubes and not simply a manifestation of the inter-nanotube junctions in the network?

Mary Chan-Park responded: It should be due to both the intrinsic properties and also affected by intertube junctions. If the tubes are not sorted, they cannot switch. Our network devices show switching of 3 to 4 orders. But the intertube resistance also affects the behaviour.

Mark Baxendale asked: From your work, is possible to comment the expected response of an FET with an individual semiconductor nanotube channel?

Mary Chan-Park answered: If we do the single tube devices, the percentage of switchable devices would correlate to the percent purity of the semiconducting tubes as determined by uv-vis spectroscopy.

Karl Coleman asked: Does the conducting performance depend on the density of the tubes?

Mary Chan-Park responded: Yes, but if there are too many tubes, the on/off ratio will be sacrificed. We typically keep the on : off ratio at the $10^{3-4}$ for comparison and that somewhat dictates the density of the tubes.

Milo Shaffer said: A range of mechanisms have been proposed to account for the selectivity of chemical reactions for different types of nanotube, including the availability of electrons at the Fermi level and the influence of bond strain. ${ }^{1}$ In your system, which do you think are the most important, and what are the implications for the competition between selection by electronic character and by diameter (or any other parameter)?

Do you titrate in the radical reagent, and if so, do you see an evolution of the selectivity with stoichiometry?

1. S. Hodge et al., Chem. Soc. Rev., 2012, 41, 4409-4429.

Mary Chan-Park responded: There are two main mechanisms - diameter dependence and chirality due to confinement of wave vector in the quasi-1D cylinders.

There are both effects but the arc discharge tubes are in a narrow diameter range of around $1.4 \mathrm{~nm}$ to $1.6 \mathrm{~nm}$. In this range, the Fermi level electron transfer to the radicals predominate in the reactions. 
It is hard to titrate the radical because they cannot be gradually controlled easily.

Rebecca Edwards remarked: I notice that there are a relatively large number of steps after the gel electrophoresis; were these steps important to the resulting properties?

Mary Chan-Park answered: Yes, the steps are mainly related to the removal of gel from the nanotube. In fact, solution spectroscopy data are relatively easier to get. To prove that devices can work, the tubes need to be sorted and they need to be cleaned. These steps are for cleaning and are very important.

Nazario Martín asked: Can you comment on the similarities and differences between the functionalisation depending on the source of the radicals? Have you tried phenyl radicals instead of naphthyl?

Mary Chan-Park replied: We tried the phenyl azo compounds before instead of the naphthyl azo compounds. They cannot differentiate the electronic structures of nanotubes.

Nazario Martín asked: Did you produce your radical in situ? They are typically quite unstable. Why did you not follow well established methodologies from diazonium salts (Tour reaction) for radical functionalisation of CNTs, graphene and fullerenes etc.?

Mary Chan-Park answered: The radicals are produced in situ by the probe sonication of azo naphthalene compounds. We have tried diazonium salts before but they are hard to control, and cannot work well for separation of large diameter nanotubes.

Karl Coleman opened the discussion of the paper by Oana Andreea Bârsan: Was the response you observed an ohmic response when you assembled your network?

Oana Andreea Bârsan responded: Our SWCNT networks showed a dominant semi-conductive behaviour with a non-ohmic response.

Karl Coleman said: When recording the electrical data from the device, did the behaviour change with addition of the polymer - did you see a different profile before and after addition of polymer?

Oana Andreea Bârsan answered: The resistance of the SWCNT films was recorded before, during, and after polymer impregnation. While blank measurements showed an increase in resistance of 3.1 times (before cooling) due to removal of dopants upon heating, all of the films showed an average increase in resistance of 4.3 times (before cooling) after the polymer impregnation process.

Mark Baxendale asked: It might be better to do impedance spectroscopy - an AC probe will give you more information given the non-ohmic response; for 
example, it is possible to extract the junction and bundle resistances, and junction capacitance.

Oana Andreea Bârsan responded: This is a very good idea. Thank you.

Sehmus Ozden asked: Are your CNTs randomly oriented or covalently interconnected?

Oana Andreea Bârsan replied: The SWCNTs we use are not functionalized so there are no covalent bonds between them.

Mary Chan-Park said: Did you anneal your sample before adding the polymer? At what temperature and for how long?

Oana Andreea Bârsan responded: The SWCNT films were subjected to a $400{ }^{\circ} \mathrm{C}$ treatment for two hours under a nitrogen atmosphere as part of the film preparation process.

Katsumi Kaneko asked: As scanning of AFM should give the SWCNT network structure, did you observe the AFM images before and after the scanning?

The information should give important nanolevel information on the stability of the network.

Oana Andreea Bârsan answered: Yes, we scanned the same area on a $~ 30 \mathrm{~nm}$ thick SWCNT film up to 5 consecutive times and we didn't observe any changes in the topography images.

Petrus Santa-Cruz said: The inhomogeneity shown in the peeled-off composite films (shown in Fig. 5 of your paper) seems to be greater than in SWCT films (Fig. 1d and $1 \mathrm{f}$ in the paper), that looks very homogeneous. What is the cause of this segregation? How does this homogeneity compare with samples prepared by traditional mixing of the SWCNTs with the uncured polymer mixture, prior to film deposition?

Oana Andreea Bârsan answered: The apparent inhomogeneity in Fig. 5 is due to the polymer that has crept in at the bottom of the SWCNTs' network during the polymer impregnation process, forming droplet areas of pure polymer. Around these areas, ropes of SWCNTs are visible sticking out of the composite film that most likely got pulled while peeling the composite off the substrate. The homogeneity at the bottom side of this composite film is not representative of the homogeneity of the SWCNTs' network inside the cured polymer matrix.

We have not prepared samples by the traditional mixing technique using exactly the same components; the high viscosity of the polymer mixture requires the addition of a solvent in the mixture and SWCNTs are extremely difficult to disperse in any type of solvent. Carbon nanotube-epoxy composites have been prepared using this technique, mostly with multi-walled CNTs, and these composites present CNTs agglomerates tens to hundreds of micrometres large (see references 10, 21, 22, 23 in the paper). 
Karl Coleman asked: Do you think the change of resistance with film thickness is real?

Oana Andreea Bârsan answered: I believe you are referring to Fig. 7c, where the polymer impregnation effect (final/initial resistance ratios) is shown as a function of SWCNT film thickness. Considering that initially, a SWCNT film $15 \mathrm{~nm}$ thin has a resistance 30 times higher than a $135 \mathrm{~nm}$ SWCNT film (Fig. 7b), it is possible that the small variations in resistance ratios after polymer impregnation seen in Fig. 7c are simply due to experimental errors. This is because each sample is unique and once the polymer film is applied on the surface of a SWCNT film and the sample is heated, it is very difficult to control the spreading of the polymer on the SWCNT film surface during the polymer impregnation process.

Pulickel Ajayan enquired: Is the discrepancy you see in the resistance of your deposited nanotube films due to varying contact resistance? Have you done four probe electrical measurements of films of different thicknesses to resolve the issue?

Oana Andreea Bârsan replied: Yes, we have measured the resistance of SWCNT films of different thicknesses using both four point probe measurements and two $\mathrm{Au}$ electrodes with a multimeter. Even if the techniques are completely different, the results are similar as you can see in Fig. $7 \mathrm{~b}$ of the paper.

Zeba Khanam asked: Why choose SWCNT instead of MWCNTs? Is there any specific reason for that?

Oana Andreea Bârsan replied: We chose SWCNTs because they have a simple structure with no inner layers and no charge transport between the inner tubes. They also have a very narrow diameter distribution (1-2 nm) and a very large aspect ratio (usually above 1000). These characteristics made them promising for our goal of preparing a uniform conductive CNT network.

Zeba Khanam commented: Its not the case that we always need a surfactant or solvent to disperse the CNTs in epoxy. The supercritical carbon dioxide treatment at high pressure enhances the dispersion even without using any dispersion agent. ${ }^{1}$

1. M. G. H. Zaidi, Modification in mechanical, thermal and electrical characteristics of epoxy through dispersion of multi-walled carbon nanotubes in supercritical carbon dioxide, Carbon Lett., 2013, 14(4), 218-227.

Karl Coleman remarked: Is your film transparent?

Oana Andreea Bârsan replied: The films' transmittance depends on their thickness (see Fig. 7a showing absorbance as a function of film thickness). The typical $\sim 30 \mathrm{~nm}$ thin SWCNT films have a transmittance of $\sim 85 \%$. 
Chris Ewels opened the discussion of the paper by Ian Kinloch: In the context of short-fibre theory and associated stress transfer, what do you think is the importance of rippling and folding in graphene in these composites?

Ian Kinloch replied: It is known in fibre composites that fibre waviness does lead to a decrease in uniaxial properties, because the waviness effectively introduces a range of fibre alignment into the system. We would expect this behaviour to be also present in graphene composites. It is also plausible that if the graphene folds such that its overall diameter is beneath the critical diameter that the flake will not provide reinforcement.

Pulickel Ajayan asked: For the multi-layered structure of graphene, is the changing Raman peak really a result of the shear between layers or due to some form of defects? Have you done the same experiment using multi-layer graphene grown by CVD? Are the results similar?

Ian Kinloch responded: We believe that the change of the Raman peak is due to the shear of the layers and this shear produces reversible stacking defects into the structure. ${ }^{1}$

1. L. Gong et al., ACS Nano, 2013, 7(8), 7287-7294.

Petrus Santa-Cruz addressed a question to both Ian Kinloch and Pulickel Ajayan: How do you control the size of the graphene flake during electrochemical exfoliation?

Pulickel Ajayan answered: The size of the exfoliated flakes depends more on the starting material (crystallite size) compared to the electrochemical conditions, in my opinion. This is different from chemical exfoliation where the flakes can be continuously reduced in size as a function of treatment conditions and time.

Ian Kinloch replied: The diameter of the graphene flakes is approximately the same as the diameter of the starting graphite particle size. The thickness of the flakes is controlled by the diameter of the starting graphite and the number of times the exfoliation process is run. We find that $5 \mu \mathrm{m}$ diameter graphite particles exfoliate to few layer graphene after 2-3 exfoliation cycles, whereas $20 \mu \mathrm{m}$ diameter graphite particles require 5 exfoliation cycles. We currently cannot exfoliate graphene with a diameter greater than $20 \mu \mathrm{m}$. Please see reference 1 for more details.

1. A. M. Abdelkader et al., ACS Appl. Mater. Interfaces, 2014, 6(3), 1632-1639.

Karl Coleman commented: If you have a mixture of flake sizes do you see that the larger flakes dominate the mechanical properties?

Ian Kinloch answered: To the first approximation, any flakes above the critical diameter will fully reinforce the composite whereas any flakes below the critical diameter will not reinforce the composite. In reality, this cut-off would be 
broader, with the degree of reinforcement dropping off as the diameter of the flake decreases beneath the critical diameter.

Pagona Papakonstantinou asked: Did your small size flakes have more oxygen than the larger flakes?

Ian Kinloch responded: We believe that the oxygen content is the same in both sized flakes and is of the order of $\sim 5$ at $\%$.

Pagona Papakonstantinou commented: It was mentioned that the amount of graphene that could be loaded is limited - would it be possible to explain or expand on this?

Ian Kinloch answered: The maximum loading of graphene, as with all reinforced composites, will be limited by a number of factors:

1) Having sufficient matrix to coat and bond the reinforcment into a coherent structure.

2) The increase of matrix viscosity with the addition of graphene making the system unprocessable.

3) Not being able to properly disperse the graphene.

Pulickel Ajayan asked: From the reinforcement point of view as a filler in composites, how do you compare a nanotube and a graphene nanoribbon of same dimensions; which do you think would provide better reinforcement?

Ian Kinloch responded: The answer to this question is not immediately obvious as there are several competing effects. The graphene nanoribbon will have a higher intrinsic modulus than the nanotube because the modulus depends on the cross-sectional area of the reinforcement and the nanotube has to account for a hole down its middle. There should also be better stress transfer for the nanoribbon since every atom its in contact with two polymer interfaces, while for the nanotube the atoms just have one side in contact with the polymer. However, the nanotubes will have a higher bending stiffness and thus persistence length than the nanoribbons due to the nanotubes' cylindrical shape and thus the nanotubes will have less waviness in the composite.

Pulickel Ajayan commented: Regarding reinforcement effects of the tubes versus ribbons: I suppose what is different is not just the effect of a central hole in nanotubes but also the exposed edges present in graphene but not in nanotubes. The interfacial chemistry between the edges and the matrix should be quite different.

Karl Coleman commented on the works of Ian Kinloch and Oana Andreea Bârsan :Which technique for producing the composite as presented in your two papers (pre-making the network or not) do you think will win out?

Oana Andreea Bârsan replied: It is difficult to compare the two techniques without a target application in mind. Both techniques are viable but for different purposes. If we consider applications such as electrostatic shielding, 
electromagnetic interference shielding etc., the typical mixing approach might win out because it requires the use of small amounts of CNTs. Mechanical properties of the composite materials can also be improved using small amounts of CNTs, which would lead to a cheaper material. On the other hand, if applications such as transistors or flexible, transparent conductors are the target, premaking the conductive network might prove to be more efficient.

Karl Coleman opened the discussion of the paper by David Zitoun: You make your own graphene oxide - what are the issues with commercial sources? Is it manganese contamination?

David Zitoun responded: You're right, chemical contamination is the main concern (especially Mn).

Karl Coleman commented: Your device seems to have more consistent cycling performance when using rGO - the capacity of the GO sample is dipping below the rGO. Is the GO turning into rGO?

David Zitoun answered: The hypothesis of an electrochemical reduction of GO to rGO makes sense at the working potential (between 0 and $1 \mathrm{~V} v s \mathrm{Li}^{+} / \mathrm{Li}$ ). Only Raman spectroscopy after cycling would give a clear answer.

Karl Coleman remarked: Should we read anything into the fact that the capacity curves for GO and rGO crossover?

David Zitoun answered: Not really, it means that some of the capacity of $\mathrm{Si}$ cannot be accessed at the beginning in the rGO (too many mechanical constraints) while GO is a much more flexible matrix. However, it is too flexible when it comes to long-term stability.

Pulickel Ajayan said: You have used a half cell. So why is the performance of the $\mathrm{Si}-\mathrm{GO}$ anode better than $\mathrm{Si}-\mathrm{rGO}$ ?

David Zitoun responded: The active material performs as the electrode versus metallic Li (half-cell) in excess. The limiting factor is still the Si which needs to be well encapsulated (Si-rGO), otherwise, some of the $\mathrm{Si}$ "delaminate".

Pulickel Ajayan asked: Is the weight the total weight of the anode reported $\mathrm{Si}$ and GO or just Si alone?

David Zitoun responded: We've reported the values normalized to Si weight; GO does not show any Li intercalation in our experiments.

Santosh Kumar Bikkarolla communicated: You have mentioned glassy carbon should be used as the counter electrode instead of platinum wire. I am wondering about the conductivity of a glassy carbon electrode due to the amorphous nature. Instead of glassy carbon electrode it will more appropriate to use graphite rod as counter electrode which is more conducting due to crystalline nature as done by 
Liang et al.. ${ }^{1}$ Can you provide any reference where a glassy carbon has been used as the counter electrode?

1. Y. Liang et. al., Nat. Mater., 2011, 10, 780-786.

David Zitoun communicated in reply: Glassy carbon is used routinely in electrochemistry. The company Pine is for instance selling rotating disc electrodes and counter electrodes. The use of GC as counter electrode is a directive from the Argonne labs in Los Alamos. However, it is fair to mention that many articles still use platinum as counter electrode.

Rebecca Edwards opened the discussion of the paper by Alexander Zöpfl: It states in your paper that you get reproducible electrical properties from your spincoated rGO films, does that include reproducibility across different batches of rGO?

Alexander Zöpfl responded: The reproducible electrical resistance is given in a range of 100-250 k $\Omega$. This range covers the resistance of different batches of reduced graphene oxide (rGO). The deviation reflects only partially properties of the material itself, it is more a result of the spin coating process. The preparation of rGO via Hummers' method followed by a reduction shows only little variance from batch to batch. In our opinion, one of the most critical influences is the starting graphite material. Here, we observed e.g. great differences between powder and flake graphite (which was used in this work) in flake size, dispersibility, and also in the Raman spectra. For our different batches from the same starting material, we could not observe changes in Raman characteristics or the final sensing behaviour which are tolerable for any practical application. To achieve highly reproducible electrical resistance was not the focus of our work. Here, we concentrated more on the optimisation of only some spin coating parameters. The substrate surface (properties, structure), the spinning solution (concentration of rGO, spreading), and the spin coating process itself (speed, acceleration, time) have been investigated. In an industrial process one has also to consider parameters like e.g. the adjustment of the substrate chips in the spin coater or the reproducible deposition of the spinning solution at identical positions, which can influence the final sensor properties.

David Zitoun commented: Do you have a way to measure the time response of your sensors? What is the role of the reduced graphene oxide in the sensor?

Alexander Zöpfl replied: The reduced graphene oxide (rGO) is used as asensor material in a chemiresistive setup. The material undergoes a very fast and high sensitive change in its electrical conductance upon gas adsorption already at low temperature $\left(25-85{ }^{\circ} \mathrm{C}\right)$. This makes it superior to conventional metal oxide sensor materials which need to be operated at higher temperatures. Reduced graphene oxide shows more p-type semiconducting behavior and therefore mainly positive charge carriers (holes) are involved. The electron transfer from rGO to adsorbed gas molecules leads to more positive charge carriers and therefore the conductance of rGO is improved, as observed e.g. for $\mathrm{NO}_{2}$. 
The thickness of the sensing layer is crucial for fast and reversible detection of gases. Therefore, a 2D material which practically consists only of a surface without any bulk phase is clearly favourable to get a fast and reversible response. Our sensor obviously consists of multiple layers which is clearly reflected in the sensors response (see e.g. Fig. SI8b in the paper) comprising of a fast and huge change in signal in the beginning and a smaller and slower change afterwards, which is a consequence of the diffusion of the analyte into the sensor layer.

David Zitoun asked: Can you synchronize your gas sensing/mixing lines with the potentiostat to measure the time response of the sensor?

Alexander Zöpfl replied: The gas mixing device and the sensing sourcemeter could be synchronized to measure the response time of the sensor. However, it is also important to know that the gas is completely mixed before it reaches the sensor. The two main parameters in this context are the size of the measurement cell and the flow rate. Many publications lack such practical information. Considering a real application, we chose a decent flow rate of $100 \mathrm{sccm}$, a relatively small gas chamber (see Fig. SI1b in the paper) and an additional mixing tube right after the mass flow controller (see Fig. SI1a in the paper). Yet, we still could observe an impact of the flow rates on the measured signal change and therefore only time responses below $1 \mathrm{~min}$ have been reported. Much shorter response times in the region of seconds have been obtained when using the sensor outside the gas chamber under ambient conditions. Due to environmental effects on the sensor setup these measurements are difficult to compare and were not mentioned in the manuscript.

David Zitoun asked: There is another mechanism of sensing with Pd based on volumetric expansion of Palladium hydride - you get the opposite response? What is your mechanism?

Alexander Zöpfl responded: The signal originates from catalytic effects of the metal nanoparticles on the graphene. Similar observations have been made for single walled carbon nanotubes and nanoribbons which were decorated with Pd. ${ }^{1,2}$ It is supposed that hydrogen atoms dissociate within Pd and lower its work function, allowing electrons to jump easily from Pd to the graphene structure. The electron transfer results in an increase of resistance and respectively a decrease in conductance because of the p-type semiconducting behaviour of reduced graphene oxide.

1. S. Mubeen, T. Zhang, B. Yoo, M. A. Deshusses, and N. V. Myung, J. Phys. Chem. C, 2007, 111(17), 6321-6327.

2. J. L. Johnson, A. Behnam, S. J. Pearton, and A. Ural, Adv. Mater., 2010, 22, 4877-4880.

Karl Coleman commented: You are analysing single component gases - what happens if you have a mixture?

Alexander Zöpfl answered: In fact, we are already dealing with a gas mixture of oxygen and nitrogen of the synthetic air carrier gas and the analyte gas. Humidity also was not excluded for the low temperature measurements. An array of 
different modified sensors is proposed to distinguish between different single component gases. The selectivity of each sensor for each gas was tested, and from the results it is expected that an addition of further analyte gases to the mixture results in a different signal pattern for all of the sensors. Data analysis by the model of principal component analysis should allow the detection of a single analyte in a complex gas mixture. This could not be tested within this study due to the limitation in the gas mixing device which only consists of three mass flow controllers.

David Zitoun opened the discussion of the paper by Santosh Kumar Bikkarolla: $99 \%$ of the literature is working on cells with acidic electrolytes - why are you using alkaline? How long do you test your materials for?

Santosh Kumar Bikkarolla responded: There is a paper Koper $^{1}$ which states: "Several reasons have emerged as to why one should prefer an alkaline electrolyte to an acidic one. Many materials are more stable in alkali."

1. M. T. M. Koper, Nat. Chem., 2013, 5, 255-256.

Rebecca Edwards remarked: Given that you are using graphene oxide (and partially reduced GO) in alkaline solutions do you think there could be an effect on the catalysis during the reaction brought about by the removal of oxidative debris from the graphene oxide surface (as described by Thomas et al. ${ }^{1}$ )?

1. H. R. Thomas et al., Chem. Mater., 2013, 25, 3580-3588.

Santosh Kumar Bikkarolla answered: In the work presented in Thomas et al., ${ }^{1}$ as well as that in Rourke et al., ${ }^{2}$ oxidation debris were successfully separated from GO by using base-washing protocols. These protocols involved either (i) treatment in weak basic solutions for long periods (more than $3 \mathrm{~h}$ ) or (ii) smaller periods assisted by heat treatment or (iii) treatment in strong basic solutions.

In our experiments the ORR performance of graphene oxide or electrochemically reduced graphene oxide was carried out on electrodes produced by drop casting ink on glassy carbon with nafion as a binder at a weak basic solution of 0.1 $\mathrm{M} \mathrm{KOH}$ solution at room temperature. Removal of oxidation debris from the surface of the electrode cannot take place under our operation conditions, however very mild deoxygenation of the electrode surface may take place only when experiments are carried out over the duration of many hours.

1. H. R. Thomas et al., Chem. Mater., 2013, 25, 3580-3588.

2. J. P. Rourke et al., Angew. Chemie Int. Ed., 20131, 50, 3173-3177.

David Zitoun commented: But the issue isn't the ORR in an alkaline cell, as shown in the review article. ${ }^{1}$

1. M. T. M. Koper, Nat. Chem., 2013, 5, 255-256.

Santosh Kumar Bikkarolla replied: We have to disagree on this point. The efficient ORR from inexpensive catalysts is a serious bottleneck in the commercialization of current fuel cells working in either acidic or alkaline environments. 
This problem is well documented in the literature and an enormous amount of research effort has been dedicated to the replacement of Pt catalysts (see references $^{1,2,3}$ ). The paper by Koper ${ }^{4}$ does not elaborate on the ORR of inexpensive catalysts.

1. Y. Liang et al., $\mathrm{Co}_{3} \mathrm{O}_{4}$ nanocrystals on graphene as a synergistic catalyst for oxygen reduction reaction, Nat. Mater., 2011, 10, 780-786.

2. J. Suntivich et al., Design principles for oxygen-reduction activity on perovskite oxide catalysts for fuel cells and metal-air batteries, Nat. Chem., 2011, 3, 546-550.

3. F. Cheng et al., Rapid room-temperature synthesis of nanocrystalline spinels as oxygen reduction and evolution electrocatalysts, Nat. Chem., 2011, 3, 79-84.

4. M. T. M. Koper, Hydrogen electrocatalyis: A basic solution, Nat. Chem., 2013, 5, 255-256.

Santosh Kumar Bikkarolla asked David Zitoun: You have mentioned that silver is a non-expensive electrocatalyst which can allow fuel cells to be commercialized. Would it be possible for you to provide some references which mention this statement? In addition can give some references which study the ORR performance of silver in fuel cell conditions in acidic media?

David Zitoun answered: There are some references describe the ORR in alkaline medium. One of the most up-to-date reviews is by Neburchilov et al. ${ }^{1}$

1. V. Neburchilov, H. Wang, J. J. Martín, W. Qu, J. Power Sources, 2010, 195, 1271.

Hitoshi Ogihara commented: The properties of rGO and GO electrocatalysts such as types of surface functional groups, electrical conductivity, and electrochemically active surface area are different. What is the most important factor effecting the performance for ORR activity of the electrocatalysts?

Pagona Papakonstantinou answered: Graphene oxide, GO (>30 at\% oxygen) is an insulator. The electrocatalyst needs to be conductive to allow efficient electron transfer for ORR. For this reason GO has low ORR activity. So conductivity is a very important factor.

One should note that in many cases, the reduction of GO is usually accompanied by nitrogen incorporation even in small amounts inherited by commonly used reduction agents (eg $\mathrm{N}_{2} \mathrm{H}_{4}, \mathrm{NH}_{3}-\mathrm{OH}$ ). Overall the reduction process reduces the work function of the material. Addition/combination of heteroatoms (oxygen is always present) helps to reduce further the work function and enhances the efficiency of ORR from $2 \mathrm{e}$ towards $4 \mathrm{e}$.

Zeba Khanam communicated: I want to know the meaning of term background signal in LSV? Kindly elaborate more with respect to ErGO.

Pagona Papakonstantinou communicated in reply: The background response is measured by taking LSVs in an $\mathrm{Ar}$ or $\mathrm{N}_{2}$ saturated solution. By subtracting this background signal from the one obtained in an $\mathrm{O}_{2}$ saturated solution we get the true ORR response of the material under investigation.

Zeba Khanam communicated: I could not understand the results of the impedance studies. The GO shows a large arc and large charge transfer resistance with higher impedance than ErGO. Why it is so? Kindly explain. 
Pagona Papakonstantinou communicated in reply: The impedance spectra mirror the ORR activity because a small bias has been applied close to the onset potential . A high ORR activity is reflected by a small semicircle which indicates a small charge transfer resistance as it allows fast shuttling of electrons during ORR. You may see this also in Fig. $9 \mathrm{~b}$ of the paper which provides the modulus of impedance $\left(\sqrt{ }\left(Z_{\text {real }}^{2}+Z_{\text {imag }}^{2}\right)\right)$ as a function of frequency. In the low frequency regime it is obvious that the modulus of $Z$ is smaller for the electrochemically reduced graphene oxide. Basics of impedance spectra can be found on the application notes of various companies specializing on EIS.

Petrus Santa-Cruz presented some slides of his groups' recent work: At the LandPhoton Group (Chemistry Department of UFPE-Brazil), we have developed a photonically-marked CNT with rare earth coordination complexes, in which the CNT act as an antenna in this system. We named the luminescent CNT as LCND, from Light Conversion Nanostructured Device.

Previously we have designed and produced photonic molecular devices by the supramolecular association of chemical species in a supramolecular way, ${ }^{1}$ leading to Light Conversion Molecular Devices (LCMD) as similar to those described by Lehn. ${ }^{2}$

For the new use of CNT as antenna in the LCND I am presenting at this meeting, we employed green chemistry methodology to functionalize in two steps, first by a click reaction assisted by ultraviolet radiation, and then, by complexation assisted by microwave irradiation. This process was presented in our poster at this meeting entitled "Photonic carbon meta-nanotubes as light conversion nanostructured devices (LCNDs)".

Here we chose the europium for the active part of the complex, and present a MWCNT-Eu ${ }^{3+}$ LCND with a better quantum efficiency than the non-hybrid complex, as shown in our poster. In addition, the final material presents excellent dispersion in water, broadening the applications for these photonically-marked CNTs, to track then in nature or biological systems, as shown in my slides.

The highly efficient photonic CNT-Eu hybrid system is produced in two steps: in the first one a previous photonic functionalization occurs with 4-Azido benzoic acid under UV-B radiation, and in a second step, the europium complex is growth under microwave irradiation. LCMD molecular devices are based on the "antenna effect" of ligands. We propose that in these our nanostructured LCND devices, the covalently functionalized carbon nanotubes may act as a nanostructured antenna, since we have experienced an enhanced luminescence quantum yield.

1. G. F. de Sá, O. L. Malta, C. Donega, A. M. Simas, R. L. Longo, P. A. Santa-Cruz, E. F. Silva, Spectroscopic Properties and Design of Highly Luminescent Lanthanide Coordination Complexes, Coord. Chem. Rev., 2000, 196, 165-195.

2. N. Sabbatini, M. Guardigli, J.-M. Lehn, Luminescent Lanthanide Complexes as Photochemical Supramolecular Devices, Coord. Chem. Rev. 1993, 123, 201-228.

Varsha Khare opened a general discussion of the topics raised at the meeting: In the discussion at this meeting, the use of carbon materials in controlling environmental pollution was hardly mentioned. As scientists, we have responsibilities to society e.g. to provide safe and clean water free from toxicity. 
Zeba Khanam communicated: As a research scholar in the Dept. of Environmental Science working with CNTs, I would be interested in the comments of the delegates on the applications of carbon nanomaterials in environmental science, in particular with reference to the papers presented at this meeting.

Pulickel Ajayan commented: We are nearly 30 years into the emergence of carbon nanomaterials, starting with the discovery of fullerenes. It seems that the field of carbon nanoscience has had a great run with a new material emerging as the savior when another wanes in popularity; such as nanotubes replacing fullerenes and graphene stealing the limelight from nanotubes. Can we predict what might be the "next kid on the block"?

Alan Windle replied: For the materials scientists and technologists, the challenge to turn nanocarbons into useful materials where they positively impact industry and thus society is really just getting underway. They are all going to be rather busy with what we have got. But what are the successors to buckyballs, CNTs and graphene ? In one sense we are probably rather running out of dimensions to do much new with carbon. One way of answering this question is to say what fascinates me in the carbon allotrope area. I think I would go unstable and start taking a deeper interest in linear acetylenic carbon (carbyne to its friends), ${ }^{1}$ which is a carbon-only chain with alternating triple and single bonds. It is of course highly unstable and predicted to exothermically cross link, perhaps explosively, which would add to the fun. Staying with instability, there are also the diamondoids (e.g. reference 2), small cage structures larger than adamantane but with an upper limit at what one might call a nano diamond crystal. They are stabilised by non-carbon additions and thus not really allotropes, but with carbon positions which tend to map onto the diamond lattice.

However, will there be another interesting, distinct carbon allotrope? Probably, but I cannot guess what, although there is an important pointer from the history of the three types of nanocarbon we currently focus on. Whatever the new form will turn out to be, it will be likely to have left calling cards for us to recognise. $\mathrm{C}_{60}$, probably the one nano allotrope which did really come from left field, had been leaving its fingerprint for decades before 1985 as an anomalously large peak at 720 amu in mass spectra on equipment which could handle such heavy molecules. Carbon nanotubes had been seen and recognised, as sub micron fibrils (the nano word had yet to be applied) almost since electron microscopes were invented, certainly since 1952. ICI ran reformers at one of their plants in North England. Regularly, they had to close the plant down, in order to scrape a strange fibrous carbon deposit from the inside of the nickel steel reaction tubes. They buried $\mathrm{kg}$ of the black stuff, so perhaps future industrial archeologists will discover a carbon nanotube deposit on the site, even mine it. The key papers which introduced the science of soot to the scientific cognoscenti were from Iijima, who in the words of Rick Smalley, definitely discovered nanotubes best, if not first. As for graphene, the molecule of graphite - if one can accept 'molecule' when its molecular weight is undefinable - its isolation using 'sellotape' was presaged by Tony Kelly (arguably the father of composite science) who used exactly that technique to prepare graphite samples thin enough so they could be observed in the TEMs of the 1960s. Perhaps he did get down to a single layer, but his interest was in interlayer dislocations. Of course, the recent Nobel prizes for graphene 
were rewards for putting everything together, and carrying our brilliant electrical measurements on graphene, and relating the results, which will be of great commercial value, to the physics derived from the Dirac, $K$ points. These three nanocarbon structures, the pinnacles of progress in carbon nanoscience, have happened because people had fun, which somehow encouraged lateral thinking the true mother of invention.

1. R. H. Baughman, Science, 2006, 312(5776), 1009.

2. M. N. R. Ashfield, P. W. May, C. A. Pego, and N. M. Everitt, Chem. Soc. Rev. 1994, $23,21$.

Sehmus Ozden remarked: Graphene nanoribbons and graphene quantum dots can be synthesized using solution chemistry. I wonder if it might be possible to synthesize carbon nanotubes via solution chemistry?

Toshiaki Enoki asked: Most of carbon nanomaterials, which have been investigated, are mostly $\mathrm{sp}^{2}$-based carbon nanomaterials. In this meeting, we discussed $\mathrm{sp}^{2}$-based carbon nanomaterials, but $\mathrm{sp}^{3}$ carbon nanomaterials should be added to the carbon nanomaterials in the future. Actually, when the size of diamond becomes nanodimensional, the structural instability is expected to give interesting phenomena. The issues surrounding this should be investigated. 